International Mathematical Forum, 2, 2007, no. 66, 3293 - 3301

\title{
A Construction of Lower-Potent Quasi-Antiorders in Semigroup with Apartness
}

\author{
Daniel Abraham Romano \\ Department of Mathematics and Informatics, Banja Luka University \\ 2, Mladen Stojanovich Street, 78000 Banja Luka, Bosnia and Herzegovina \\ bato49@hotmail.com
}

\begin{abstract}
In the present paper, we describle a construction of lower-potent positive quasi-antiorder in semigroup with apartness.

Mathematics Subject Classification: Primary 03F65; Secondary: 20M35, 20M99
\end{abstract}

Keywords: Constructive mathematics, coequality, anti-congruence, antiorder, quasi-antiorder, positive quasi-antiorder, lower-potent quasi-antiorder

\section{Preliminaries and Introduction}

This investigation, in Bishop's constructive mathematics in sense of well-known books [1, 2], [5] and Romano's papers [7 - 10], is continuation of forthcoming Crvenkovic and Romano's paper [3], and Romano's papers [11, 12]. Bishop's constructive mathematics is develop on Constructive Logic (or Intuitionistic Logic ([15])) - logic without the Law of Excluded Middle $P \vee \neg P$. Let us note that in Constructive Logic the 'Double negation Law',

$$
P \Longleftrightarrow \neg \neg P
$$

does not hold, but the following implication

$$
P \Longrightarrow \neg \neg P
$$

holds even in Minimal Logic.

Let $(S,=, \neq)$ be a set. The relation $\neq$ is a binary relation on $S$, which satisfies the following properties:

$$
\neg(x \neq x),
$$




$$
\begin{gathered}
x \neq y \Longrightarrow y \neq x, \\
x \neq z \Longrightarrow x \neq y \vee y \neq z, \\
x \neq y \wedge y=z \Longrightarrow x \neq z .
\end{gathered}
$$

Following Heyting, it called apartness. Let $Y$ be a subset of $S$ and $x \in S$. Following Bridges, by $x \bowtie Y$ we denote $(\forall y \in Y)(y \neq x)$ and by $Y^{C}$ we denote subset $\{x \in S: x \bowtie Y\}$ - the strong compliment of $Y$ in $S$. The subset $Y$ of $S$ is a strongly extensional ([13]) in $S$ if and only if $y \in Y \Longrightarrow y \neq x \vee x \in Y$.

A relation $q$ on $S$ is a coequality relation on $S$ if and only if it is consistent, symmetric and cotransitive $([9])$. Let $(S,=, \neq, \cdot)$ be a semigroup with apartness. A relation $\tau$ on $S$ is a quasi-antiorder ([10.11]) on $S$ if

$$
\begin{aligned}
\tau & \subseteq \neq \\
(\forall x, y, z \in S)((x, z) \in \tau & \Longrightarrow(x, y) \in \tau \vee(y, z) \in \tau)
\end{aligned}
$$

It $\tau$ is a quasi-antiorder on $S$ compatible with the semigroup operation in $S$, then ([11]) the relation $q=\tau \cup \tau^{-1}$ is an anticongruence on $S$. Firstly, the relation $q^{C}=\{(x, y) \in S \times S:(x, y) \bowtie q\}$ is a congruence on $S$ compatible with $\mathrm{q}$, in the following sense $q \circ q^{C} \subseteq q$ and $q^{C} \circ q \subseteq q$ ([9], Theorem 1). We can construct the semigroup $S /\left(q^{C}, q\right)=\left\{a q^{C}: a \in S\right\}$ ([9], Theorem 2) with $a q^{C}=b q^{C} \Longleftrightarrow(a, b) \bowtie q, a q^{C} \neq b q^{C} \Longleftrightarrow(a, b) \in q, a q^{C} \cdot b q^{C}=(a b) q^{C}$.

We can also construct the semigroup $S / q=\{a q: a \in S\}$ ([9], Theorem 3) with

$$
a q=b \Longleftrightarrow(a, b) \bowtie q, a q \neq b \Longleftrightarrow(a, b) \in q, a q \cdot b q=(a b) q
$$

It is easily to establish the isomorphism $S /\left(q^{C}, q\right) \cong S / q$.

A subset $A$ of a semigroup $S$ is consistent if for $a, b$ of $S a b \in A$ implies $a \in A$ and $b \in A$. it is easily to check if $A$ is a consistent subset of $S$, then $A^{C}$ is a subsemigroup of $S$. Opposite assertion, "If $T$ is a subsemigroup of $S$, then $T^{C}$ is a consistent subset of $S$." not holds in general. A consistent subsemigroup $F$ of $S$ will called a filter of $S$. In that case, the subset $F^{C}$ is a completely prime ideal of $S$. The opposite assertion "If $\mathrm{J}$ is a completely prime ideal of $S$, then $J^{C}$ is a filter of $S . "$ not holds in general.

In the Classical Semigroup Theory concept of positive quasi-order has been introduced by B.M.Schein. After that, positive quasi-orders have been studied from different points of view by many authors, mainly by T.Tamura [13-14], M.S.Putcha [6], and S.Bogdanovic and M.Ciric [4]. Quasi-antiorder relation in semigroups with apartness the first time defined by this author in his one earlier paper in 1996. Further investigation on basic properties of quasi-antiorder relations on sets and semigroups with apartness the author has did in his paper [10] and in forthcoming papers [11, 12]. Positive quasi-antiorders studied by Crvenkovic and Romano in their forthcoming paper [3]. 
For undefined notions and notations we referee to books $[1-2,5]$ and to papers $[7-10]$.

In this paper we study a constructive aspect of construction of positive quasi-antiorders of semigroups with apartness.

\section{Positive Quasi-antiorders}

By a quasi-antiorder we mean a consistent cotransitive relation on a set. For a quasi-antiorder $\sigma$ on a semigroup $S$ we say that it is compatible with the semigroup operation if and only if

$$
(\forall a, b, x \in S)(((a x, b x) \in \sigma \Longrightarrow(a, b) \in \sigma) \wedge((x a, x b) \in \sigma \Longrightarrow(a, b) \in \sigma)) .
$$

A relation $\tau$ on a semigroup $S$ is called positive if and only if

$$
(\forall a, b \in S)((a, a b) \bowtie \tau \wedge(b, a b) \bowtie \tau),
$$

and it is called lower-potent if

$\left(a^{n}, a\right) \bowtie \tau$, for any $a \in S$ and any $n \in \mathbf{N}$.

Clearly, if $\tau$ is a quasi-antiorder compatible with the semigroup operation, then it is lower-potent if and only if $\left(a^{2}, a\right) \bowtie \tau$, for all $a \in S$. Indeed: Suppose that $\left(a^{2}, a\right) \bowtie \tau$ for any $a \in S$ holds and suppose that $\left(a^{k}, a\right) \bowtie \tau$, for any $a \in S$ and $k \in \mathbf{N}$, and let $(u, v)$ be an arbitrary element of $\tau$. Then:

$(u, v) \in \operatorname{tau} \Longrightarrow\left(u, a^{k+1}\right) \in \tau \vee\left(a^{k+1}, a^{2}\right) \in \tau \vee\left(a^{2}, a\right) \in \tau \vee(a, v) \in \tau$

$\Longrightarrow u \neq a^{k+1} \vee\left(a^{k}, a\right) \in \tau \vee\left(a^{2}, a\right) \in \tau a \neq v$

$\Longrightarrow\left(a^{k+1}, a\right) \neq(u, v) \in \tau$.

So, by induction, we have that the formula $\left(a^{n}, a\right) \bowtie \tau$ holds for any $a \in S$ and any $n \in \mathbf{N}$.

In the following we will describe a construction of maximal positive quasiantiorder relation on a semigroup $S$. Let $a$ and $b$ be elements of $S$. Then, by Theorem 6 in [9], the set $C_{(a)}=\{x \in S: x \bowtie S a S\}$ is a consistent subset of $S$ such that:

(i) $a \bowtie C_{(a)}$;

(ii) $C_{(a)} \neq \emptyset \Longrightarrow 1 \in C_{(a)}$;

(iii) let $a$ be an invertible element of $S$. Then $C_{(a)}=\emptyset$;

(iv) $(\forall x, y \in S)\left(C_{(a)} \subseteq C_{(x a y)}\right)$;

$(\mathrm{v}) C_{(a)} \cup C_{(b)} \subseteq C_{a b}$.

The consistent subset $C_{(a)}$ is called a principal consistent subset of $S$ generated by $a$. We introduce relation $f$ on $S$, defined by $(a, b) \in f$ if and only if $b \in C_{(a)}$. The relation $f$ has the following properties ([9], Theorem 7):

(a) $f$ is a consistent relation on $S$;

(b) $(a, b) \in f \Longrightarrow(\forall x, y \in S)((x a y, b) \in f)$; 
(c) $(a, b) \in f \Longrightarrow(\forall n \in \mathbf{N})\left(\left(a^{n}, b\right) \in f\right)$;

(d) $(\forall x, y \in S)((a, x b y) \in f \Longrightarrow(a, b) \in f)$;

(e) $(\forall x, y \in S) \neg((a, x a y) \in f)$.

For an element $a$ of a semigroup $S$ and for $n \in \mathbf{N}$ we introduce the following notations

$$
\begin{aligned}
& A_{n}(a)=\left\{x \in S:(a, x) \in^{n} f\right\}, \\
& A(a)=\{x \in S:(a, x) \in c(f)\} \\
& B_{n}(a)=\left\{y \in S:(y, a) \in^{n} f\right\}, \\
& B(a)=\{y \in S:(y, a) \in c(f)\} .
\end{aligned}
$$

In the following two lemmas we will present some basic characteristic of these sets.

Lema 2.1 Let $a$ and $b$ be elements of a semigroup $S$. Then:

(1) The set $A(a)=\cap_{n \in N}$ is the maximal strongly extensional consistent subset of $S$ such that $a \bowtie A(a)$.

(2) $A(a) \cup A(b) \subseteq A(a b)$.

Proof immediately follows from Theorem 2 and Theorem 3 of the paper [10].

Symmetrically, we have:

Lemma 2.2 Let $a$ and $b$ be elements of a semigroup $S$. Then:

(1) The set $B(a)=\cap_{n \in N^{B}} B_{n}(a)$ is the maximal strongly extensional ideal of $S$ such that $a \bowtie B(a)$

(2) $B(a b) \subseteq B(a) \cap B(b)$.

Proof immediately follows from Theorem 4 and Theorem 5 of the paper [10].

In the next theorem we give a construction of the maximal positive quasiantiorder relation in semigroup $S$.

Theorem 2.3 The relation $c(f)$ is the maximal positive quasi-antiorder relation on semigroup $S$.

Proof immediately follows from Lemmas 2.1 - 2.2 and Lemma 3.2 in [3].

Theorem 2.4 A quasi-anti-order $\tau$ on a semigroup $S$ is positive ifand only if it is contained in the maximal quasi-antiorder relation $c(f)$ on $S$.

Proof: It is clear that if $\tau$ is a positive quasi-antiorder relation on $S$, then $\tau \subseteq c(f)$, since $c(f)$ is the maximal positive quasi-antiorder relation on $S$.

Let $\tau \subseteq c(f)$. Then, $(x, x y) \bowtie c(f) \supseteq \tau$ and $(x, y x) \bowtie c(f) \supseteq \tau$ for any $x, y$ of $S$. So, the quasi-antiorder $\tau$ is positive.

Concluding note I: As mentioned in the Theorem 2.4, a quasi-antiorder on a semigroup $S$ is positive if and only if it is contained in the maximal 
positive quasi-antiorder $c(f)$. So, the family of positive quasi-antiorders on $S$ is a principal ideal of the family $\boldsymbol{Q}(S)$ of all quasi-antiorders on $S$ gneerated by the relation $c(f)$ on $S$.

Besides, we will describe maximal lower-potent positive quasi-antiorder relation on a semigroup $S$. Let $S$ be a semigroup with apartness and $a, b$ arbitrary elements of $S$. As mentioned above in this section, the set $C_{(a)}$ is a consistent subset of $S$ called a principal consistent subset of $S$ generated by the element a. Set $\operatorname{cr}\left(C_{(a)}\right)=\left\{x \in S:(\forall n \in \mathbf{N})\left(x^{n} \in C_{(a)}\right)\right\}$ is called a coradical of principal consistent subset of $S$ generated by the element $a$. We introduce a relation $s$ on $S$, on the following way

$$
(a, b) \in s \Longleftrightarrow b \in \operatorname{cr}\left(C_{(a)}\right)
$$

and we will describe some properties of relations $s$ and $c(s)$

Lemma 2.5 ([12], Lemma 2.1) The relation $c(s)$ satisfies the following properties :

(1) $c(s)$ is a consistent relation on $S$;

(2) $c(s)$ is a cotransitive relation ;

(3) $(\forall n \in \mathbf{N})\left(\left(a, a^{n}\right) \bowtie c(s)\right)$.

For an element $a$ of a semigroup $S$ and for $n \in \mathbf{N}$ we introduce the following notations:

$$
\begin{aligned}
& A_{n}(a)=\left\{x \in S:(a, x) \in^{n} s\right\}, \\
& A(a)=\{x \in S:(a, x) \in c(s)\} \\
& B_{n}(a)=\left\{x \in S:(x, a) \in^{n} s\right\}, \\
& B(a)=\{x \in S:(x, a) \in c(s)\} .
\end{aligned}
$$

By the following results we will present some basic characteristics of these sets.

Lemma 2.6 ([12], Theorem 2.3) Let $a$ and $b$ be elements of a semigroup $S$. Then:

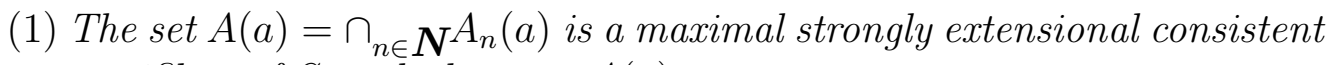
potent semifilter of $S$ such that $a \bowtie A(a)$.

(2) $A(a) \cup A(b) \subseteq A(a b)$;

(3) $(\forall n \in \mathbf{N})\left(A(a)=A\left(a^{n}\right)\right)$

Analogously, we have:

Lema 2.7 ([12], Theorem 2.3) Let $a$ and $b$ be elements of a semigroup $S$. Then:

(1) The set $B(a)=\bigcap_{n \in N^{B}} B_{n}(a)$ is the maximal strongly extensional completely potent semiprime ideal of $S$ such that $a \bowtie B(a)$. 
(2) $B(a b) \subseteq B(a) \cap B(b)$;

(3) $(\forall n \in \mathbf{N})\left(B\left(a^{n}\right)=B(a)\right)$.

Theorem 2.8 The relation $c(s)$ is the maximal positive quasi-antiorder relation on a semigroup $S$ and the following $(\forall a \in S)(\forall n \in \mathbf{N})\left(\left(a^{n}, a\right) \bowtie c(s)\right)$ holds.

textbfProof immediately follows from Lemmas 2.6 - 2.7 and lemma 3.2 in [3].

Theorem 2.8 A positive quasi-antiorder $\tau$ on a semigroup $S$ is lowerpotent positive if and only if it is contained in the maximal lower-potent positive quasi-antiorder on $S$.

Proof: It is clear that if $\tau$ is a lover-potent positive quasi-antiorder relation on $S$, then $\tau \subseteq c(s)$, since $c(s)$ is the maximal lower-potent positive quasiantiorder on $S$.

Let $\tau \subseteq c(s)$. Then $\left(x^{n}, x\right) \bowtie c(s) \supseteq \tau$ for any $x$ of $S$ and for any natural number $n$. So, the positive quasi-antiorder $\tau$ is lower-potent.

\section{Concluding note II:}

(1) it is clear that the family $Q_{l p}(S)$ of lower-potent positive quasi-antiorders on $S$ is completely lattice. Indeed: Let $\left\{\tau_{k}\right\}_{k \in J}$ beb a family of lower-potent positive quasi-antiorders on a semigroup $S$. Since $(\forall k \in J)(\forall n \in \mathbf{N})\left(\tau_{k} \bowtie\right.$ $\left.\left(a^{n}, a\right)\right)$, we have $\cup_{k \in J} \tau_{k} \bowtie\left(a^{n}, a\right)$. besides, $c\left(\cap_{k \in J} \tau_{k}\right)$ is a positive quasiantiorder in $S$, and the following

$$
c\left(\cap_{k \in J} \tau_{k}\right) \subseteq \cap_{k \in J} \tau_{k} \bowtie\left(a^{n}, a\right)
$$

folds for any natural $n$. So, $Q_{l p}(S)$ is a completely lattice.

(2) As mentioned in the Theorem 2.9, a positive quasi-antiorder on a semigroup $S$ is lower-potent positive quasi-antiorder relation on $S$ if and only if it is contained in the maximal lower-potent positive quasi-antiorder $c(s)$. So, the family $Q_{l p}(S)$ of lower-potent positive quasi-antiorders on $S$ is a principal ideal of $Q_{p}(S)$ generated by the relation $c(s)$ on $S$.

\section{The Main Results}

In the paper [14], Tamura turned a general method for construction of new relations from a given relation. Namely, to any relation $\rho$ on a semigroup $S$ he associated the relation $a(\rho)$ on $S$ defined by $(a, b) \in a(\rho) \Longleftrightarrow(\exists n \in$ $\mathbf{N})\left(\left(a, b^{n}\right) \in \rho\right)$, and the transitive closure of $a(\rho)$. Expect in the Tamura's paper, such constructed relations have been intensively used in a series of papers by M.S.Putcha.

Using this ideal, in this paper for a given relation $\sigma$ in a semigroup $S$ with apartness we construct the following relation: 


$$
(a, b) \in p(\sigma) \Longleftrightarrow(\forall n \in \mathbf{N})\left(\left(a, b^{n}\right) \in \sigma\right) .
$$

A sense of such method is to build a lower-potent quasi-antiorder from a given relation $\sigma$. namely, the following assertion can be easily proved:

Theorem 3.1 The maximal lower-potent quasi-antiorder on a semigroup $S$ contained in $\sigma$ on $S$ equals $c p(\sigma \cap \neq)$.

Proof: It is clear that $c p(\sigma \cap \neq)$ is a consistent and cotrasitive in semigroup $S$. By [7], $c p(\sigma \cap \neq)$ is the maximal quasi-antiorder relation under $\sigma \cap \neq$. let $u, v$ and $a$ be arbitrary elements of $S$ such that $(u, v) \in c p(\sigma \cap \neq)$. Then:

$$
\begin{aligned}
& (u, v) \in c p(\sigma \cap \neq) \Longrightarrow\left(u, a^{n}\right) \in c p(\sigma \cap \neq) \vee\left(a^{n}, a\right) \in c p(\sigma \cap \neq) \vee(a, v) \in \\
& c p(\sigma \cap \neq)
\end{aligned}
$$

for any natural $n$. Thus, for any natural $n$, we have:

$(u, v) \in c p(\sigma \cap \neq) \Longrightarrow u \neq a^{n} \vee\left(a^{n}, a\right) \in c p(\sigma \cap \neq) \vee a \neq$

$\Longrightarrow(u, v) \neq\left(a^{n}, a\right) \vee(\forall m \in \mathbf{N})\left(\left(a^{n}, a^{m}\right) \in(\sigma \cap \neq)\right)$

$\Longrightarrow\left(a^{n}, a\right) \neq(u, v) \in c p(\sigma \cap \neq)$.

Let $\tau$ be a positive quasi-antiorder under $\sigma$. Then $\tau \subseteq \neq$ and $\tau \subseteq c(\sigma)$. Thus, $\tau \subseteq \sigma \cap \neq$. Suppose that $\tau \subseteq^{n}(\sigma \cap \neq)$ for some $n \in \mathbf{N}$. Hence,

$$
\tau \subseteq \tau * \tau \subseteq(\sigma \cap \neq) *^{n}(\sigma \cap \neq)=^{n+1}(\sigma \cap \neq) .
$$

So, by induction, we have $\tau \subseteq \cap_{n \in \mathbf{N}}^{n}(\sigma \cap \neq)$. Therefore, the relation $c p(\sigma \cap \neq)$ is the maximal lower-potent quasi-antiorder under $\sigma \cap \neq$.

On the other hand, a good characteristic of this construction is that it supplies positiveness property, so the following theorem can be easily verified:

Theorem 3.2 The maximal lower-potent positive quasi-antiorder on a semigroup $S$ contained in a relation $\sigma$ on $S$ equals $c p(\sigma \cap f)$

Proof: Let $(u, v)$ ne an arbitrary element of $c p(\sigma \cap f)$ and let $a, b$ be elements of $S$. Then:

$(u, v) \in c p(\sigma \cap f) \Longrightarrow(u, a) \in c p(\sigma \cap f) \vee(a, a b) \in c p(\sigma \cap f) \vee(a b, v) \in$ $c p(\sigma \cap f)$

$\Longrightarrow u \neq a \vee(a, a b) \in c p(\sigma \cap f) \vee a b \neq v$

$\Longrightarrow(a, a b) \neq(u, v) \in c p(\sigma \cap f)$.

For $(b, a b) \bowtie c p(\sigma \cap f)$ the proof is analogous. So, $c p(\sigma \cap f)$ is a positive quasi-antiorder under $\sigma$.

Let $\tau$ be a positive quasi-antiorder under $\sigma$. Then, $\tau \subseteq c(f)$ and $\tau \subseteq c(\sigma)$. Thus, $\tau \subseteq \sigma \cap f$. Suppose that $\tau \subseteq^{n}(\sigma \cap f)$ for some $n \in \mathbf{N}$. Hence,

$$
\tau \subseteq \tau * \tau \subseteq(\sigma \cap f) *^{n}(\sigma \cap f)=^{n+1}(\sigma \cap f)
$$


So, by induction, we have $\tau \subseteq \cap_{n \in \mathbf{N}}^{n}(\sigma \cap f)$. Therefore, the relation $c p(\sigma \cap f)$ is the maximal positive quasi-antiorder under $\sigma$.

Therefore, if we start from a consistent and positive relation $\tau$, then withouth serious problems we obtain that $c p(\tau)$ is a positive lower-potent quasiantiorder. But, if we want to obtain that $c p(\tau)$ is compatible with the semigroup operation, then more serious difficulties are arising. Namely, there is a following construction

$$
c p(\tau)^{+}=\left\{(a, b) \in S \times S:\left(\exists u, v \in S^{1}\right)((u a v, u b v) \in c p(\tau))\right\}
$$

-a minimal extension of $c p(\tau)$ - such that it is compatible with the semigroup operation, but with losing of substantial characteristics. So, the problem of compatibility of relation $c p(\tau)$ with the semigroup operation is open.

\section{References}

[1] E. Bishop: Foundation of Constructive Analysis; McGraw-Hill, New York 1967.

[2] D.S. Bridges and F. Richman: Varieties of Constructive Mathematics; London Mathematical Society Notes 97, Cambridge University Press, Cambridge 1987.

[3] S. Crvenkovi'c and D.A. Romano: On Positive Quasi-antiorders in Semigroups with Apartness, pp. 1-15, (To appear).

[4] S. Bogdanović and M. Ćirić: Semigroups; Prosveta, Niš, 1993 (In Serbian).

[5] R. Mines, F. Richman and W. Ruitenburg: A Course of Constructive Algebra; Springer, New York 1988.

[6] M.S. Putcha: Positive Quasi-order on Semigroups; Duke Math. J., 40(1073), 857-869.

[7] D.A. Romano: On Construction of Maximal Coequality relation and its Applications; In: Proceedings of 8th international conference on Logic and Computers Sciences "LIRA '97", Novi Sad, September 1-4, 1997, (Editors: R.Tosic and Z. Budimac), Institute of Mathematics, Novi Sad 1997, 225-230.

[8] D.A. Romano: A Left Compatible Coequality Relation on Semigroup with Apartness; Novi Sad J. Math., 29(2), 221-234.

[9] D.A. Romano: Some Relations and Subsets of Semigroup with Apartness Generated by Principal Consistent Subsets; Univ.Beograd, Publ. Elektroteh. Fak. Ser. Math., 13(2002), 7-25.

[10] D.A. Romano: Construction of Classes of the Quasi-antiorder Relation Generated by Principal Consistent Subsets on Semigroup with Apartness; In: 
Proceedings of 16th Conference on Applied Mathematics; Budva, Monte Negro, May 31 - June 04, 2004 (Editors: N. Krejic and Z.Luzanin), Department of Mathematics, University of Novi Sad, Novi Sad 2006, 129-135.

[11] D.A. Romano: A Note on Quasi-antiorder in Semigroup; Novi Sad J. Math., 37(1)(2007), 1-6.

[12] D.A. Romano: Quasi-antiorder on Semigroup Generated by Coradical of Principal Consistent Subsets; 1-9 pp, (Submited in a journal at 01.02.2007)

[13] T. Tamura: Semilattice Congruences viewed from Quasi-orders; Proc. Amer. Math. Soc., 41(1973), 75-79.

[14] T. Tamura: Quasi-orders, Generalized Archimedeanss, Semilattice Decompositions; Math. Nachr. 68(1975), 201-220.

[15] A.S. Troelstra and D. van Dalen: Constructivism in Mathematics, An Introduction, Volume II; North-Holland, Amsterdam 1988.

\section{Received: June 23, 2007}

